\title{
Effect of Jet Vortex Generators on Shock Wave Induced Separation on Gas Turbine Profile
}

\section{FLASZYNSKI Pawel ${ }^{*}$, DOERFFER Piotr, PIOTROWICZ Michal}

\author{
Szewalski Institute of Fluid-Flow Machinery, Polish Academy of Sciences, Fiszera 14, PL 80-231, Gdansk, Poland
}

(C) The Author(s) 2021

\begin{abstract}
The interaction between a shock wave and a boundary layer on a suction side of gas turbine profile, namely Transition Location Effect on Shock Wave Boundary Layer Interaction, was one of main objectives of TFAST project. A generic test section in a transonic wind tunnel was designed to carry out such investigations. The design criteria were to reproduce flow conditions on the profile in wind tunnel as the one existing on the suction side of the turbine guide vane. In this paper, the effect of film cooling and jet vortex generators on the shock wave boundary layer interaction and shock induced separation is presented. Numerical results for Explicit Algebraic Reynolds Stress Model with transition modeling are compared with experimental data.
\end{abstract}

Keywords: shock wave, gas turbine, flow control, jet vortex generators

\section{Introduction}

In the last decades, great attention has been paid and development has been done to maintain laminar boundary layer in many applications [1-3]. This research mostly concerned laminar wings, with particular focus on maintaining laminar boundary layer on the longest possible distance. The research on the laminar boundary layer is immanently coupled with the investigations of instabilities and disturbances leading to laminar-turbulent transition.

In the recent High Pressure (HP) gas turbine stages, highly loaded aerofoils are applied. In such cascades, transonic and even supersonic flow zones exist. High pressure turbine vanes are usually film cooled and coolant is blew from the holes trips boundary layer. However, due to the strong acceleration on the suction side, boundary layer is relaminarised farther downstream of cooling holes rows, which has a significant effect on the shock induced separation. As heat transfer and film cooling effectiveness are of crucial importance in highly loaded turbine stages, an understanding of transition mechanisms and its location is required for a competitive product.

The transition location effect on the shock wave boundary layer interaction, namely Transition Location Effect on Shock Wave Boundary Layer Interaction, was one of the objectives of the TFAST EU project. Such interaction on highly loaded gas turbine profile with film cooling was the objective of one work package. Additionally, an investigation of jet vortex generators application upstream of the shock wave to trip boundary layer and to reduce the separation was also carried out.

Flow structure in turbomachinery cascades, particularly if applied film cooling, is complex and the application of (air) jet vortex generators (AJVG) requires careful consideration. AJVG for low pressure turbine have been tested experimentally and numerically and published in Ref. [4, 5]. An aerothermal impact of AJVG in a linear turbine cascade under the influence of film cooling at different Mach and Reynolds numbers is presented in Ref. [6]. 


\begin{tabular}{llll}
\hline \multicolumn{2}{l}{ Nomenclature } & \multicolumn{2}{l}{ x cartesian coordinate $/ \mathrm{m}$} \\
AJVG & air jet vortex generators & $x$ & Greek symbols \\
$c$ & chord $/ \mathrm{m}$ & $\delta$ & boundary layer thickness $/ \mathrm{mm}$ \\
FC & film cooling & $\delta^{*}$ & displacement thickness $/ \mathrm{mm}$ \\
$H$ & shape factor & $\delta^{* *}$ & momentum thickness $/ \mathrm{mm}$ \\
$P_{\mathrm{c}}$ & total pressure (coolant plenum) & $\rho$ & density $/ \mathrm{kg} \cdot \mathrm{m}^{-3}$ \\
$P_{0}$ & total pressure (inlet) & $\rho_{\infty}$ & density at boundary layer edge $/ \mathrm{kg} \cdot \mathrm{m}^{-3}$ \\
$v$ & velocity $/ \mathrm{m} \cdot \mathrm{s}^{-1}$ & & \\
$v_{\infty}$ & velocity at boundary layer edge $/ \mathrm{m} \cdot \mathrm{s}^{-1}$ & & \\
\hline
\end{tabular}

\section{Profile and Test Section}

To carry out investigations of the shock wave boundary layer interaction on suction side of gas turbine profile in IMP PAN wind tunnel, a single passage test section was designed. One should note that, for selected case, the shock wave interacts with boundary layer on suctions side only. Due to that, in order to reproduce the boundary layer on the profile as the one existing in the cascade, pressure distribution on the suction side was the design criteria of the test section (Fig. 1). All investigations were carried out on the lower profile, while the upper profile plays an important role in creation of required pressure distribution and the wake downstream. Such test section is implemented in the wind tunnel, where cascade measurements are not available. As it is single passage test section and the inlet and outlet are designed for the selected flow conditions, a variation of operating conditions and profile loading are not possible.

Details of the test section are shown in Fig. 1. Upstream of the profiles, the inlet duct is designed in order to maintain required Mach number $\sim 0.17$. The cascade configuration was defined by TFAST Partner (Rolls-Royce Deutschland). The outlet pressure is adjusted by the movable upper wall downstream of profiles. The location and shape of the upper limiting wall influence on the pressure distribution on suction side and the outlet Mach number, which is an important parameter required for the defined conditions. For the investigated configuration, the outlet Mach number is 1.05 , which corresponds with highly loaded case.

An advantage of the simplified test section at IMP PAN is the measurement accessibility. The nozzle guide vane configuration enables following investigations:

(1) basic configuration without film cooling-location of natural transition;

(2) effect of film cooling on transition location and shock wave boundary layer interaction;

(3) tripping devices effect on shock wave boundary layer interaction.

The cascade configuration includes 2 rows of cooling holes and is defined as following:

(1) profile chord: $112.5 \mathrm{~mm}$;

(2) axial chord: $52 \mathrm{~mm}$;

(3) blade span: $100 \mathrm{~mm}$ (wind tunnel width);

(4) pitch to axial chord: $\sim$;

(5) hole diameter: $1.35 \mathrm{~mm}$ (both rows);

(6) hole distance to hole diameter: 2.5 .

In the paper, the effect of (A)JVG (Air Jet Vortex Generators) on the shock wave boundary layer interaction and the proposed model approaches for such configurations are presented.

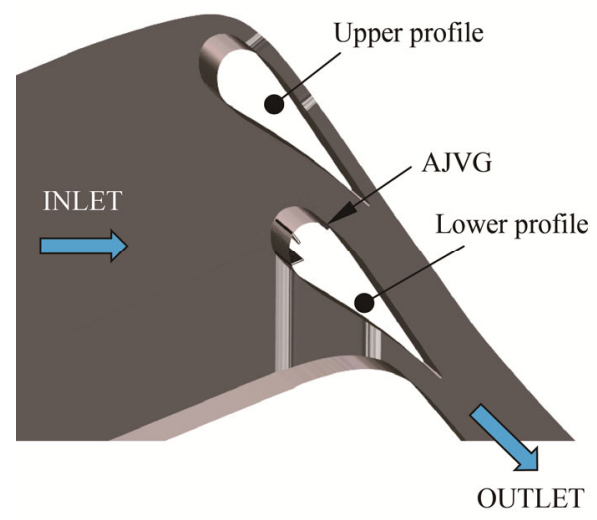

Fig. 1 Test section

\section{Numerical Model Description}

Numerical simulations are carried out by means of Fine/Turbo Numeca. Details of the turbine profile with film cooling rows and jet vortex generators are presented in Fig. 2. The structured computational mesh is generated in IGG/Numeca for 3 cases:

(1) Reference one: no film cooling and no Air Jet Vortex Generators (no FC-no AJVG);

(2) With film cooling and no Air Jet Vortex Generators (FC-no AJVG);

(3) With film cooling and with Air Jet Vortex Generators (FC-AJVG).

Computational mesh size for all cases is presented in Table 1, while the mesh topology and refinement zones 
are presented in Fig. 3. In Fig. 3(a), the topology and mesh blocks distribution for the reference configuration is highlighted. In the case of other two cases, including film cooling jet holes the general structure is similar, except the zones in the vicinity of these holes and downstream of them on the profile suction side. The mesh is refined close to the wall in order to keep $y^{+}$ below 1 (average value is 0.47 ) on the profile surface. The number of cells in boundary layer is in the range

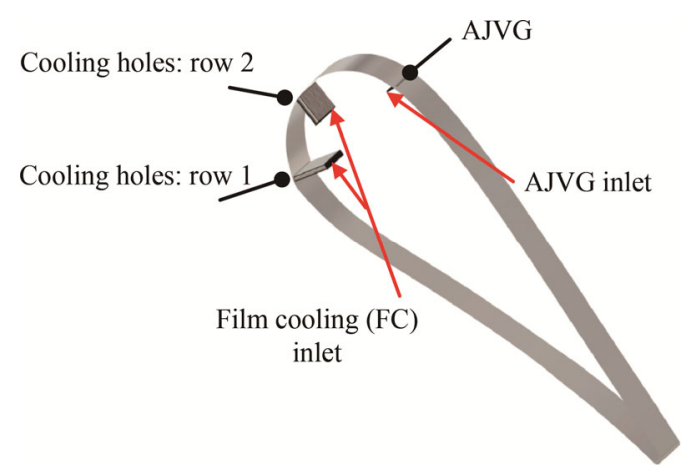

Fig. 2 Turbine profile

Table 1 Mesh details

\begin{tabular}{cccc}
\hline & no FC-no AJVG & FC-no AJVG & FC-AJVG \\
\hline No. of blocks & 34 & 825 & 1120 \\
No. of 3D cells & $9 \times 10^{6}$ & $27.5 \times 10^{6}$ & $34 \times 10^{6}$ \\
\hline
\end{tabular}

(a)

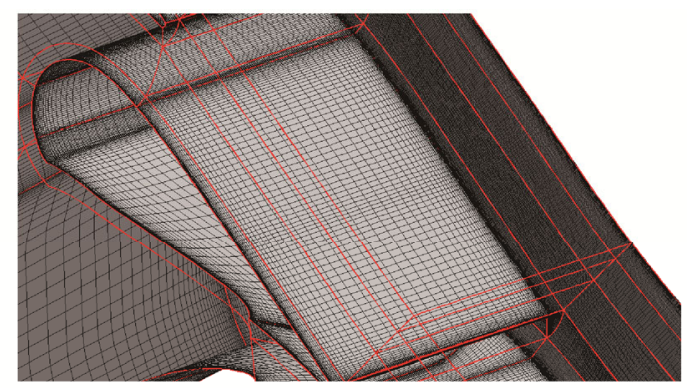

(b)

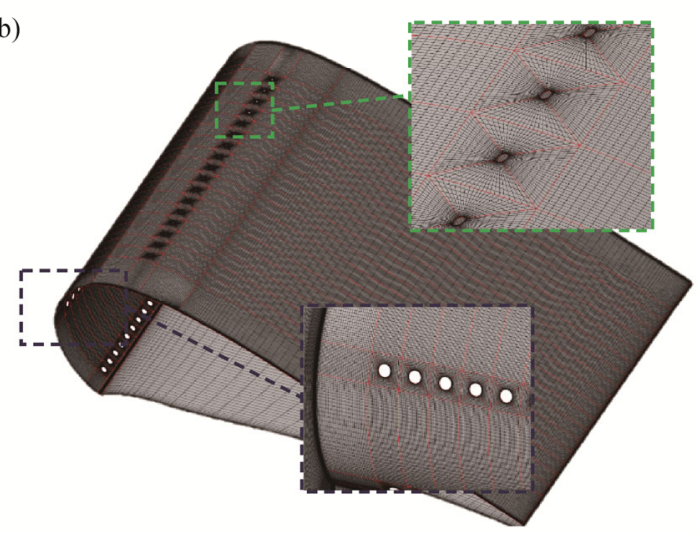

Fig. 3 Computational mesh topology (a) and details close to FC and AJVG holes (b) of 35 to 40 , while the expansion ratio is 1.15 . In the case of configurations with film cooling and AJVG, butterfly topology is used in the holes and 25 cells inside holes and 25 cells between them in spanwise direction.

Boundary conditions are set according to the measurement data. Inlet and outlet boundary conditions are listed in Table 2. At the inlet of cooling holes, the total pressure is adjusted according to the required pressure ratio. At the AJVG inlet, the total conditions are the same as in the main flow. At the outlet, static pressure is set to obtain the outlet Mach number 1.05. At the cooling holes inlet, total pressure is set according to the required pressure ratio. In the paper, results are shown for the total pressure ratio 1.05 . In the case of the jet vortex generators holes, the total conditions are applied as in the main flow. In all cases, the total temperature is the same as in the main flow and one should point out that thermal effects are not investigated. It means that the total temperature in the main flow, coolant and jet vortex generators in test section is the same. At all walls, adiabatic conditions are applied.

Table 2 Inlet and outlet boundary condition

\begin{tabular}{cc}
\hline \multicolumn{2}{c}{ INLET Main } \\
\hline Total pressure & $101 \mathrm{kPa}$ \\
Total temperature & $293 \mathrm{~K}$ \\
Turbulence intensity (all inlets) & $0.8 \%$ \\
Viscosity ratio (all inlets) & 10 \\
\hline INLET Film Cooling holes (FC) \\
\hline Total pressure & $106.05 \mathrm{kPa}$ \\
Total temperature & $293 \mathrm{~K}$ \\
\hline \multicolumn{2}{c}{ INLET AJVG } \\
\hline Total pressure \\
Total temperature \\
\hline OUTLET \\
\hline Static pressure \\
\hline
\end{tabular}

Spatial discretization using 2nd order central difference scheme with scalar artificial dissipation formulated by Ref. [8] is applied. The results presented in the paper are obtained for the two-equation nonlinear eddy viscosity turbulence model EARSM (Explicit Algebraic Reynolds Stress Model) [9] with the generalized transition model proposed by Langtry and Menter [10]. The transition model is based on the two transport equations for intermittency and transition momentum thickness Reynolds number.

\section{Numerical Model Validation}

As the first step, numerical simulations for the configuration without film cooling (no FC) and vortex generators (no AJVG) as tripping device are carried out. 
In the paper, selected experimental data presented in Ref. [11] are shown as the reference for numerical simulations.

Numerical results, shown as isentropic Mach number at the middle section of the profile (Fig. 4), indicate correct Mach number prediction upstream of the shock wave. Predicted shock wave is located slightly upstream of the measured location. It is also shown that the isentropic Mach number (pressure) distribution within the $\lambda$-foot and shortly downstream of the shock differs to the measured distribution. The more steep decrease of isentropic Mach number in the $\lambda$-foot zone is caused by its lower size than measured and due to that the pressure rises on a shorter distance. The measured and predicted shock wave location is shown also in Fig. 5, where schlieren and density gradient magnitude is presented. It is clearly visible that shock wave predicted numerically is located upstream of the measured one. Besides, the wake of upper profile and shock structure downstream of the lower profile can be noticed in both cases.

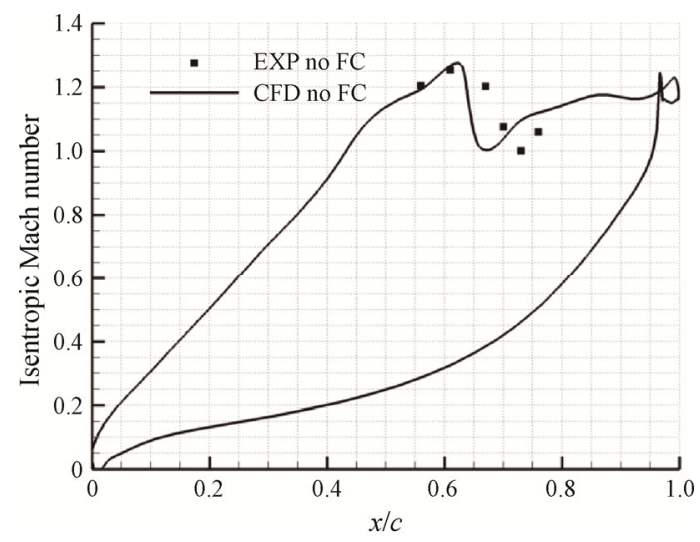

Fig. 4 Isentropic Mach number at midspan

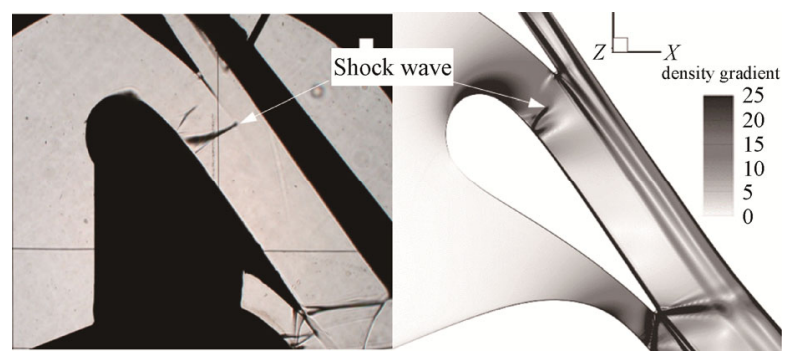

Fig. 5 Schlieren (left: EXP) [11] and density gradient magnitude (right: CFD)

As the effect, the separation bubble size downstream of the shock is slightly underpredicted by the numerical simulations. In Fig. 6, the separation is visualized by iso-surface of negative component of velocity in streamwise direction. Upstream and downstream of the blue zone, the dashed lines indicate the location of separation and reattachment detected experimentally. The length of separation bubble predicted numerically is 7 $\mathrm{mm}$, shorter than measured one which is $9 \mathrm{~mm}$.

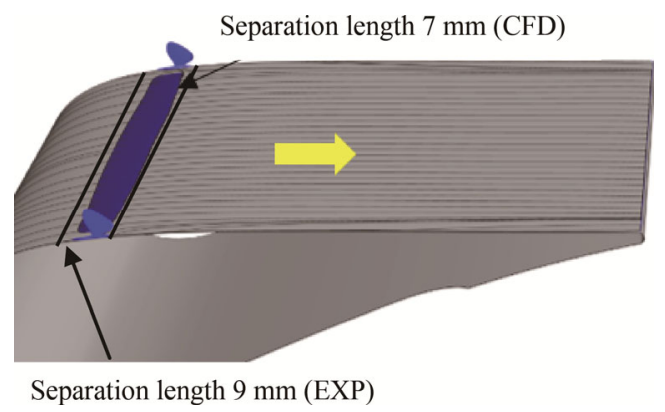

Fig. 6 Separation length visualized by iso-surface of negative velocity (no FC-no AJVG)

The differences probably arise from the different boundary layer distribution upstream of the shock wave. Boundary layer profile comparison is not available due to the measurement difficulties in very thin boundary layer $\sim 0.3 \mathrm{~mm}$. Additionally, the upstream boundary layer is laminar, which influences on the poor seeding distribution within this zone and makes Laser Doppler Anemometry measurements unavailable. Boundary layer profile measured and predicted for clean case is shown in Fig. 10. There is some discrepancy in the middle part of boundary layer, but its thickness and distribution close to the wall show good agreement.

In spite of the difference in the separation size prediction, the shown model can be applied to qualitative investigations of the film cooling and jet vortex generators effect on shock induced separation.

\section{Jet Vortex Generators Effect on Boundary Layer Separation}

Shock wave boundary layer interaction and induced separation on the suction side of turbine blade is affected by the existing film cooling. Coolant affects strongly the boundary layer distribution and finally on the interaction.

The isentropic Mach number comparison for 3 cases (with/without FC and AJVG) at the middle span is shown in Fig. 7. The pressure disturbance at location of $15 \%$ of axial chord downstream of the leading edge, is caused by the $2^{\text {nd }}$ row of cooling holes. One can notice the second difference in Mach number distribution at $55 \%$ of axial chord, where the air jet vortex generator affects the local pressure. As a consequence of film cooling and streamwise vortices generated by jets upstream of the shock wave, its interaction with the modified boundary layer is also altered.

Following figures (Figs. 8 and 9) show results for the cases with film cooling and vortex generators. Due to the existence of film cooling on the suction side and the 


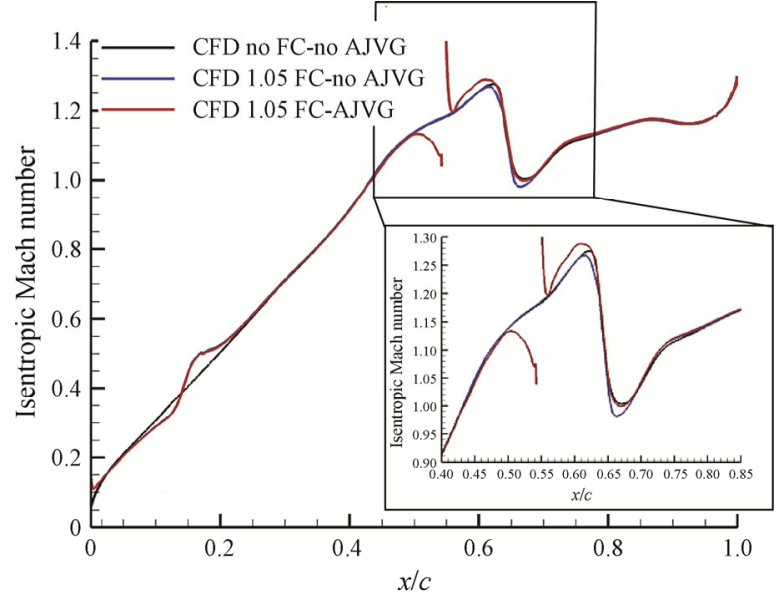

Fig. 7 Isentropic Mach number at midspan with $\mathrm{FC}$ and AJVG effect effect on the boundary layer development, a $\lambda$-foot size varies with upstream boundary layer state. As presented by numerical schlieren plots, the $\lambda$-foot size for clean case is higher than that in the other cases. In clean case (no FC and no AJVG), the shock wave interacts with laminar boundary layer. At relatively low Mach number upstream of the shock $(M=1.26)$ and laminar boundary layer, a large $\lambda$-foot is created.

One can notice the disturbance generated by the cooling holes in the middle image (Fig. 8), visualised by the increased locally density gradient magnitude.

The $\lambda$-foot size is not affected significantly if jet vortex generators are applied and no difference between cases "FC-no AJVG" and "FC-AJVG" is noticed. Some effect of AJVG is visualised by a density distribution upstream of shock wave, where the jet location is clearly seen.

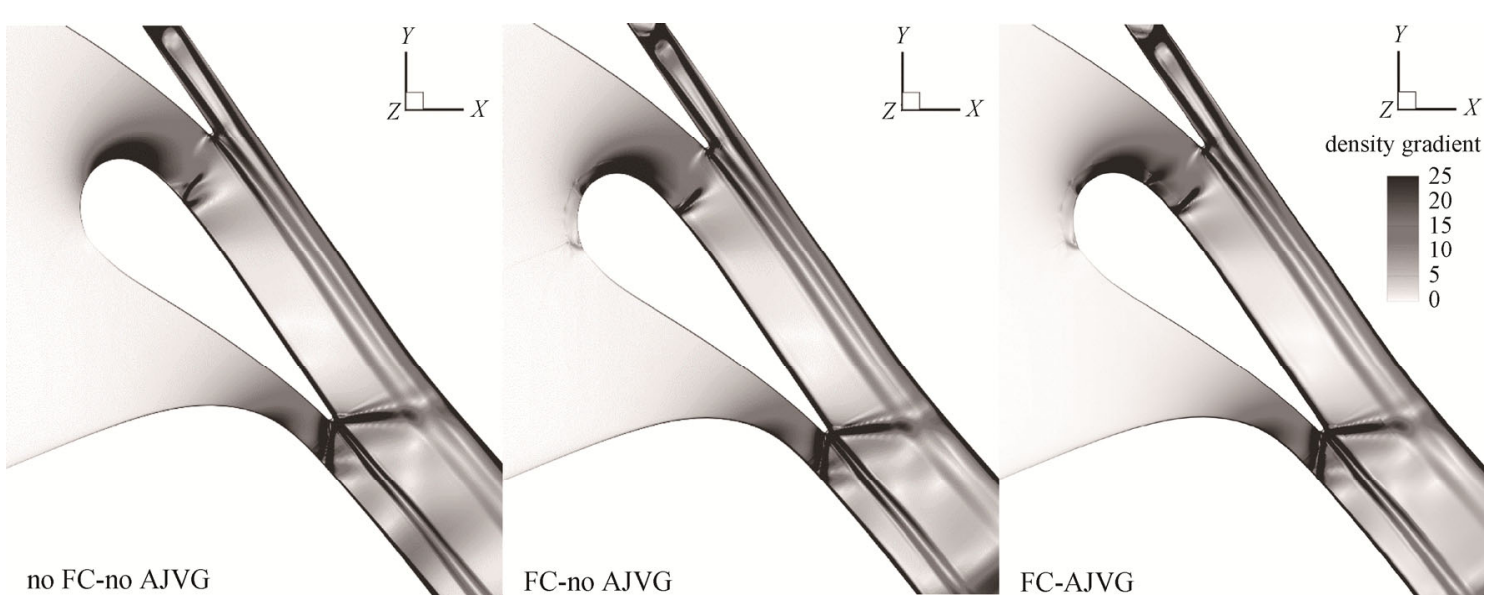

Fig. 8 Shock wave structure visualized by density gradient [11]
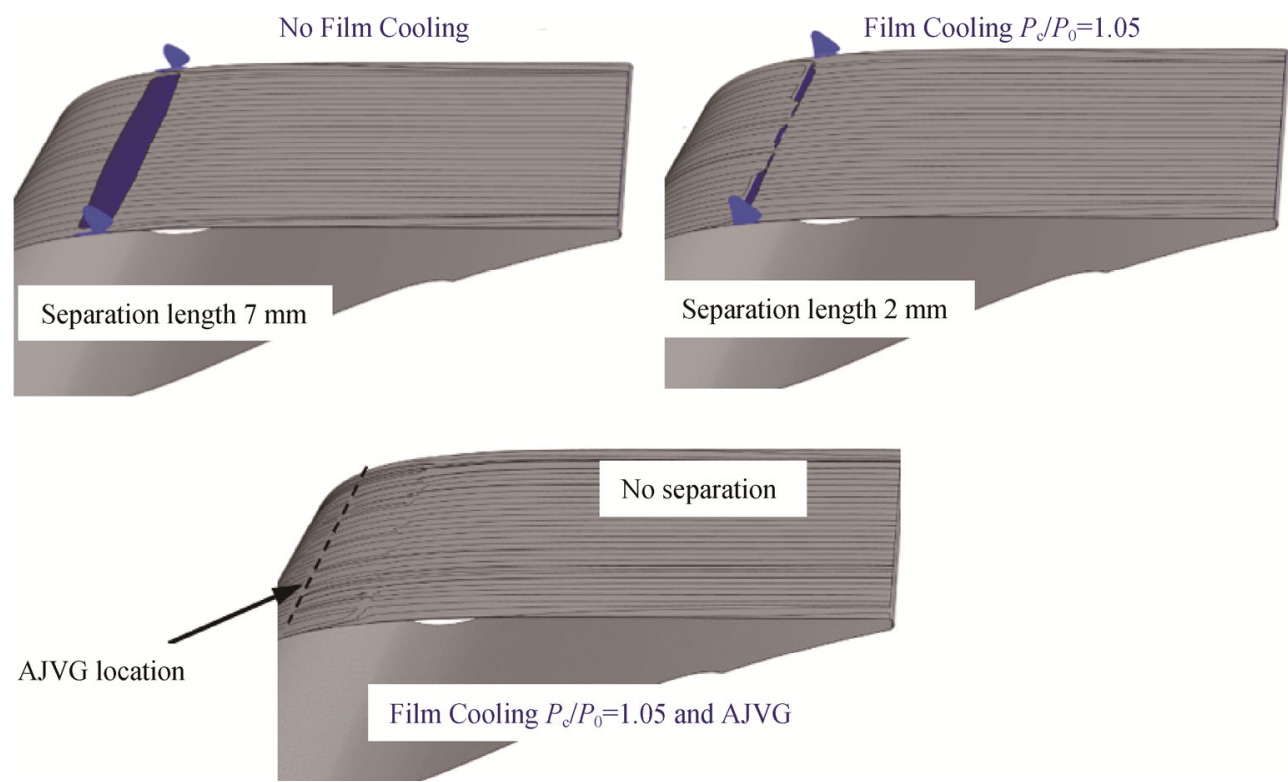

Fig. 9 Separation visualized by iso-surface of negative velocity [11] 
The $\lambda$-foot height is reduced as well as the separation length downstream of the shock wave due to the effect of film cooling and generated streamwise vortices on the upstream boundary layer. The separation reduction is shown in Fig. 9 by the iso-surface of negative streamwise velocity and streamlines near to the profile surface. As presented above, the separation length for the clean case (no FC-no AJVG) is $7 \mathrm{~mm}$. Application of film cooling for pressure ratio 1.05 leads to reduction of the separation length to $2 \mathrm{~mm}$. Such positive effect is caused by energizing of boundary layer which alters velocity profile close to the wall and increases shear stresses upstream of the shock wave.

The applied jet vortex generators lead to the creation of 3D flow structures [12] upstream of the shock and further boundary layer modification. As a consequence, the separation downstream of shock wave is removed and the flow is fully attached. This is positive effect which has to be investigated also experimentally, but it was not done within a framework of TFAST project. Anyway, numerically predicted trend of the effect of streamwise vortices on the shock wave boundary layer interaction is consistent with the other results published by the authors [13]. An important flow feature, influencing on the loss coefficient of the cascade, is the wake width. For the investigated configuration it is not possible to compare the wakes for different configurations due to the applied test section geometry. As shown in Fig. 8, shock wave generated at the trailing edge is reflected from the suction side, so the wake is affected by this reflection. In order to overcome this problem and compare velocity distribution far downstream of the shock wave, the boundary layer on the suction side, upstream of the trailing edge is presented. In Fig. 10, velocity profile for 3 cases is shown together with Laser Doppler Anemometry (LDA) measurements for clean case. One can notice that application of film cooling influences on the increase of boundary layer thickness, while the additionally applied jet vortex generators do not lead to further boundary layer changes at this location. The effect of the film cooling and generated streamwise vortices on boundary layer upstream of the trailing edge $(x / c=0.94)$ is represented in Table 3 by integral parameters:

$$
\begin{gathered}
\delta^{*}=\int_{0}^{\delta}\left(1-\frac{\rho v}{\rho_{\infty} v_{\infty}}\right) \mathrm{d} y \\
\delta^{* *}=\int_{0}^{\delta} \frac{\rho v}{\rho_{\infty} v_{\infty}}\left(1-\frac{v}{v_{\infty}}\right) \mathrm{d} y \\
H=\frac{\delta^{*}}{\delta^{* *}}
\end{gathered}
$$

All parameters prove existing effect of film cooling on boundary layer far downstream of the shock wave and very weak effect of additional vortices created by AJVG. As it is mentioned above, in the presented single passage test section there is no possibility to investigate various operating conditions or aerodynamic performance of cascade. The presented results were considered as the first step of investigations and cascade measurements in DLR Goettingen [11].

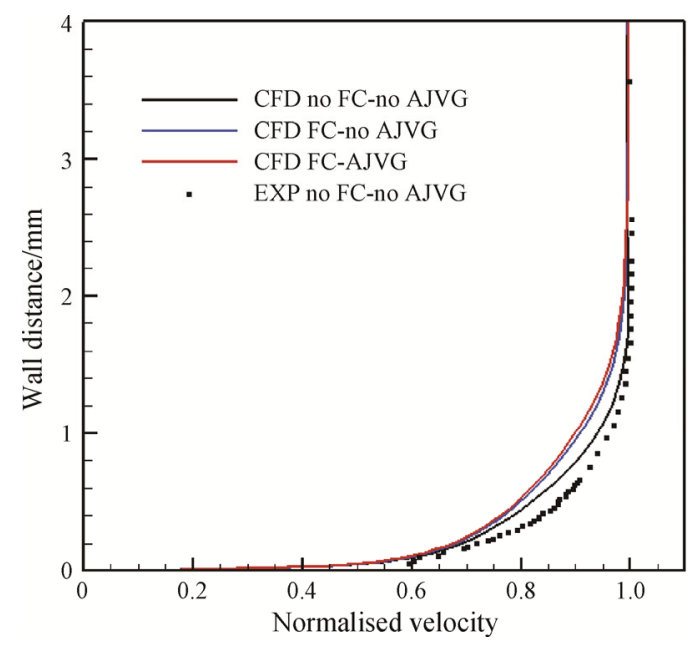

Fig. 10 Boundary layer profile at $x / c=0.94$

Table 3 Boundary-layer integral parameters at $x / c=0.94$

\begin{tabular}{cccc}
\hline symbol & $\begin{array}{c}\text { no FC-no } \\
\text { AJVG }\end{array}$ & $\begin{array}{c}1.05 \text { FC-no } \\
\text { AJVG }\end{array}$ & 1.05 FC-AJVG \\
\hline$\delta / \mathrm{mm}$ & 1.47 & 1.76 & 1.79 \\
$\delta^{*} / \mathrm{mm}$ & 0.142 & 0.145 & 0.147 \\
$\delta^{* *} / \mathrm{mm}$ & 0.063 & 0.067 & 0.068 \\
$H$ & 2.253 & 2.164 & 2.162 \\
\hline
\end{tabular}

\section{Full Span vs "Slice" Model}

AJVG influence on the separation reduction downstream of the shock wave was investigated for the full span configuration. Such approach is highly recommended if all three-dimensional effects should be taken into account. However, the configuration including film cooling holes and jet vortex generators is computationally demanding, especially if the jet holes diameter is low and a large number of jets are required. Below, results for the two approaches are shown: full span configuration and simplified one, limited to only one film cooling hole at each row and one AJVG hole. It means that the simulations are done only for the slice of the full domain. In this case at side surfaces, instead of wall, periodic boundary conditions are applied. Such approach is effective if the influence of AJVG streamwise location on the shock wave interaction is investigated.

Mach number at the middle plane for full span and 
simplified geometry with and without AJVG is shown in Fig. 11 and Fig. 12, respectively. Isentropic Mach number on profile is compared in Fig. 13. In the case of

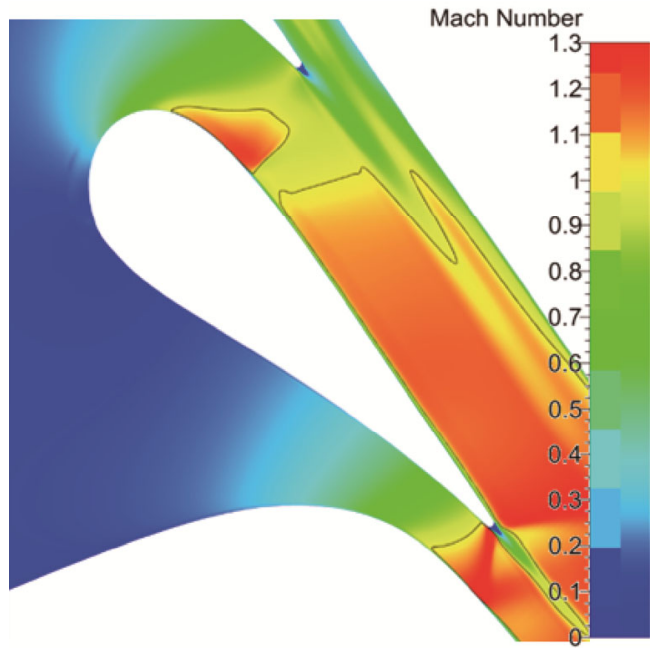

Fig. 11 Mach number at midspan: full span model

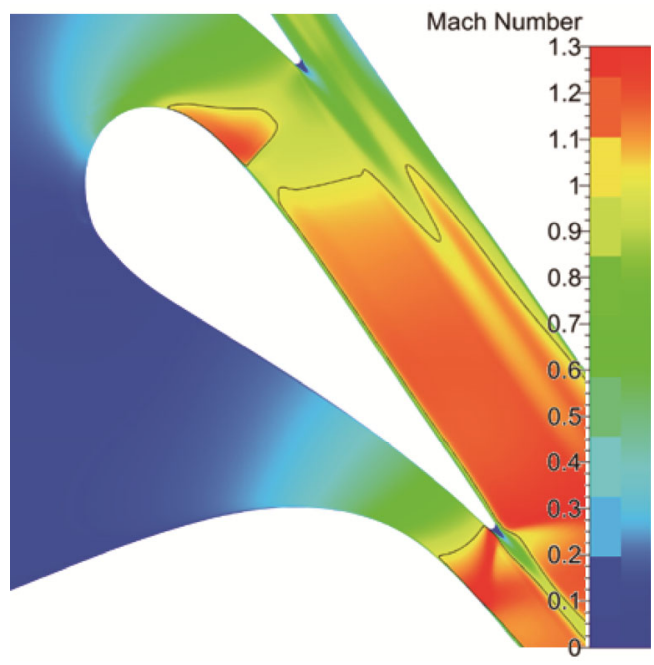

Fig. 12 Mach number at midspan: "slice" model "slice" model, the shock wave is slightly moved upstream, but the difference is insignificant. Downstream of the shock, pressure distribution is the same for both approaches.

In Figs. 14 and 15, shear stress for these configurations is compared. The "slice" model results are shown as multiplication of the single computational domain including one hole. The numerical simulations for this configuration are carried out for the domain marked by black solid lines and spanwise dimension is indicated in both figures.

If no AJVG case is considered, one can see low shear stress zone downstream of the shock wave. Results for "slice" model configuration indicate an existence of the small separation bubble, identified by negative shear stress. In the case of full span model, the value of shear stress is also negative, but it is slightly higher and it is not clearly displayed as for the "slice" approach. Additionally, sidewalls effect and the secondary flow traces are seen in the first case.

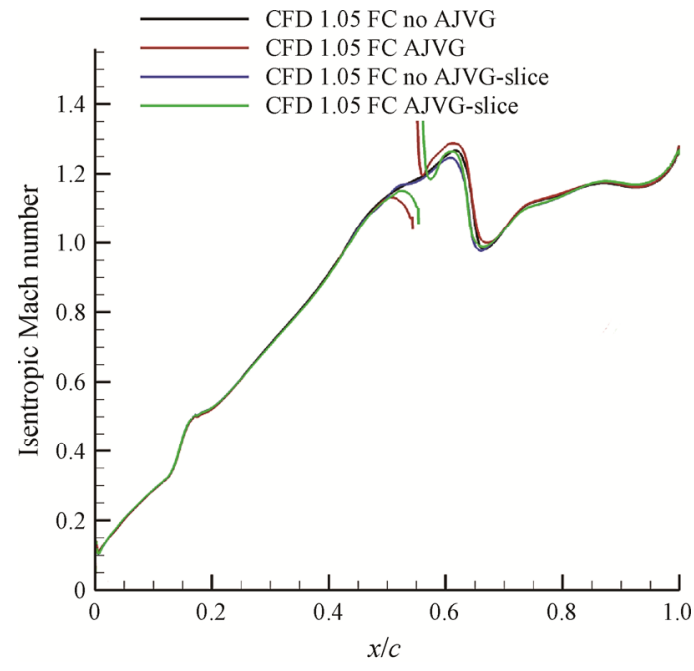

Fig. 13 Isentropic Mach at midspan: sidewalls effect

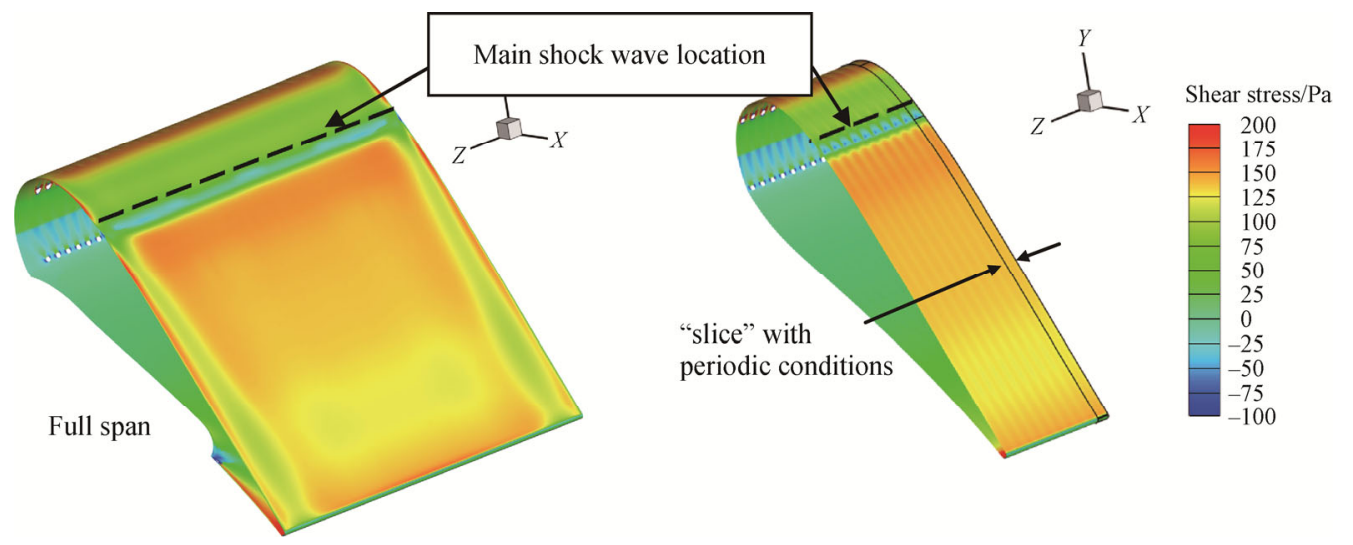

Fig. 14 Shear stress on suction side: FC-no AJVG 


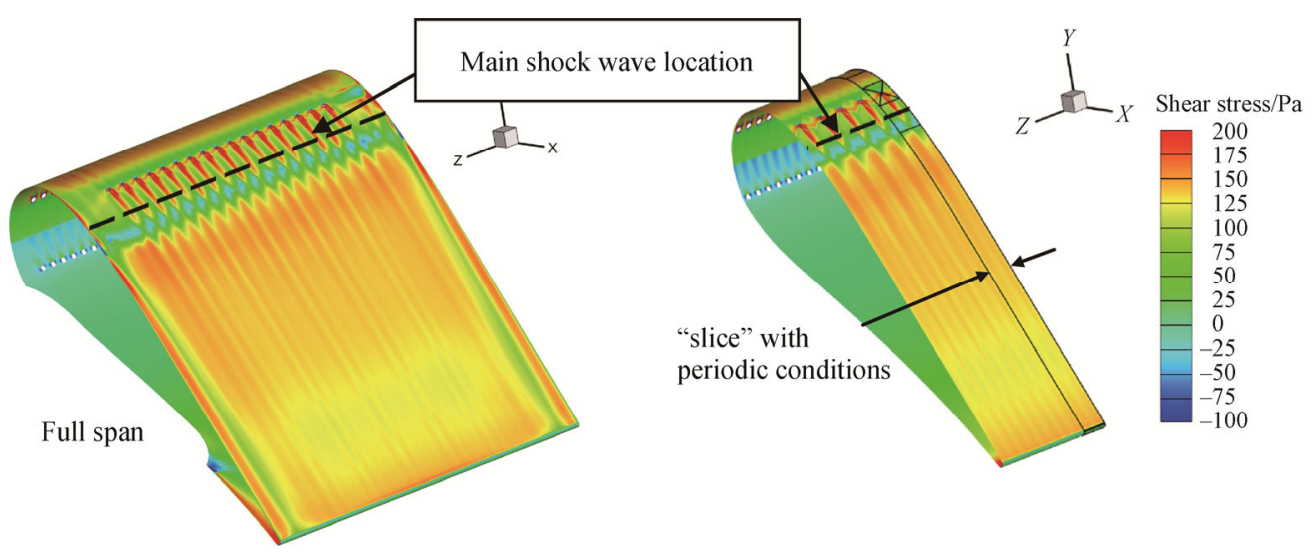

Fig. 15 Shear stress on suction side: FC-AJVG

Application of AJVG upstream of the shock wave leads to the increased shear stress and the separation is reduced in both cases, full span and periodic model (Fig. 14). Effect of streamwise vortices is strong enough to be noticed on the shear stress distribution in the interaction zone. Distribution of streamwise component of shear stress on the suction side at the middle spanwise location is shown in Fig. 16. At the chordwise location $x / c=\sim 0.5$, the shear stress increase is caused by generated streamwise vortices (red). The effect on shear stress distribution can be also noticed even if the AJVG is not activated, but the existence of the small holes influences the local velocity disturbance. At the location of shock wave boundary layer interaction, the clear impact of generated streamwise vortices is seen. Streamwise component of shear stress is positive along the wall, which indicates lack of boundary layer separation at this zone.

Further downstream, at the traverse located upstream of the trailing edge $(x / c=0.94)$, the velocity distribution in boundary layer is not dependent on the applied model and no significant differences can be noticed (Fig. 17).

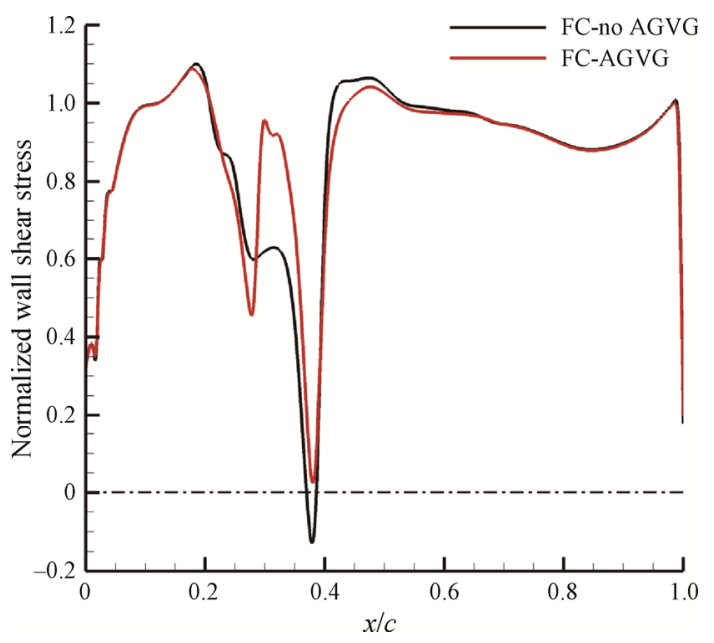

Fig. 16 Shear stress distribution on suction side

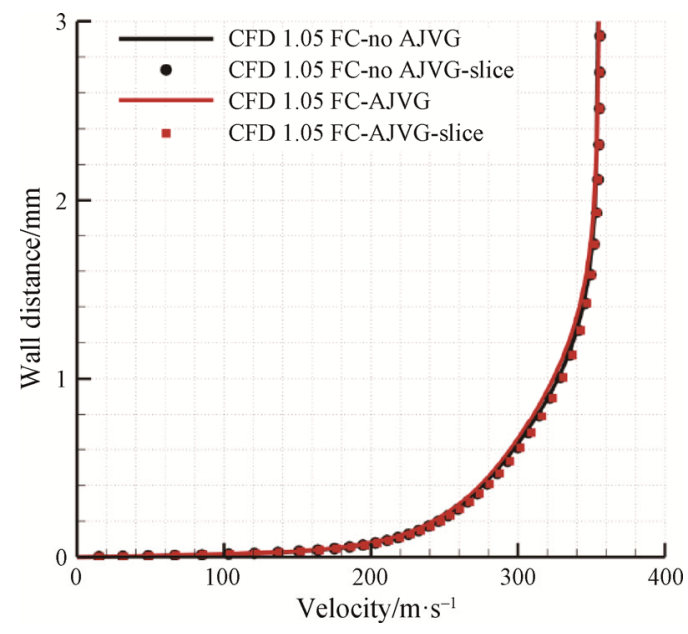

Fig. 17 Velocity profile at $x / c=0.94$

It is important observation, because the numerical simulations for AJVG on the investigated profile can be done by means of simplified model.

\section{Conclusions}

In the paper, results of numerical simulations carried out within a framework of European Project TFAST are presented. The single passage test section for investigations of shock wave boundary layer interaction on gas turbine profile was designed and the selected experimental data are considered as the reference for numerical simulations. Simulations have been carried out by means of Fine/Turbo Numeca code for 3 cases:

(1) Clean: no film cooling and no Air Jet Vortex Generators;

(2) With film cooling and without Air Jet Vortex Generators;

(3) With film cooling and with Air Jet Vortex Generators.

The comparison of numerical results with experimental data for the clean case indicates good 
agreement and possibility application of the presented approach to qualitative investigations of the AJVG effects on the shock induced separation.

AJVG influence on the separation reduction downstream of the shock wave was investigated for the full span configuration. As shown, application of film cooling on the nozzle guide vane profile causes velocity profile modification upstream of the shock wave and leads to the significant reduction of the separation downstream of the shock wave.

Numerical simulations for the configuration including cooling holes and AJVG show that applied jets located downstream of the cooling holes introduce streamwise vortices influencing on the flow structure upstream of the shock wave. As the effect, no separation bubble exists downstream of the shock wave. It proves that the separation can be reduced or removed if the streamwise vortex generator is applied. The presented results were considered as the first step of investigations and cascade measurements in DLR Goettingen.

Based on the comparison of the full span and "slice" approach, one can conclude that the simplified configuration including one pitch of the holes (with periodic boundary conditions) can be successfully applied to jet vortex generator investigations, optimization of the location or jet configuration.

\section{Acknowledgements}

This research was supported by the 7 EU framework project and was carried out within the research project with the acronym TFAST (Transition Location Effect on Shock Wave Boundary Layer Interaction).

This research was supported by CI TASK and in part by PL-Grid Infrastructure.

\section{References}

[1] Ackeret J., Feldmann F., Rott N., Investigations of compression shocks and boundary layers in gases moving at high speed. NACA (National Advisory Committee for Aeronautics Collection) Technical Memorandums, Report No. NACA-TM-1113, 1947.

URL: http://hdl.handle.net/2060/20030063988.

[2] Arnal D., Délery J., Laminar-turbulent transition and shock wave/boundary layer interaction. RTO AVT Lecture Series on "Critical Technologies for Hypersonic Vehicle Development”, held at the von Kármán Institute, Rhode-St-Genèse, Belgium, May 10-14, 2004, and published in RTO-EN-AVT-116.

[3] Davidson T., Babinsky H., Influence of transition on the flow downstream of normal shock wave-boundary layer interactions. 54 ${ }^{\text {th }}$ AIAA Aerospace Sciences Meeting, Jan 4-8, 2016, San Diego, California, USA.

https://doi.org/10.2514/6.2016-0044.

[4] Volino R., Kartuzova O., Ibrahim M., Experimental and computational investigations of low-pressure turbine separation control using vortex generator jets. Proceeding of ASME Turbo Expo, 2009.

DOI: $10.1115 /$ GT2009-59983.

[5] Ibrahim M., Kartuzova O., Volino R., LES and URANS computational investigations of LPT blade (L1A) separation control using vortex generator jets. ICHMT Digital Library Online, 2009.

DOI: 10.1615/ICHMT.2009.TurbulHeatMassTransf.1910.

[6] Gomes R., Niehuis R., Aerothermodynamics of a high-pressure turbine blade with very high loading and vortex generators. ASME Turbo Expo 2010: Power for Land, Sea, and Air, June 14-18, 2010, Glasgow, UK, 2010. Paper No. GT2010-23543.

[7] Flaszynski P., Doerffer P., Szwaba R., Kaczynski P., Piotrowicz M., Shock wave boundary layer interaction on suction side of compressor profile in single passage test section. Journal of Thermal Science, 2015, 24: 510-515.

[8] Jameson A., Schmidt W., Turkel E., Numerical solution of the Euler equations by finite volume methods using Runge Kutta time stepping schemes. 14th Fluid Plasma Dyn. Conf., American Institute of Aeronautics and Astronautics, Reston, Virigina, 1981. DOI: 10.2514/6.1981-1259.

[9] Menter F.R., Garbaruk A.V., Egorov Y., Explicit algebraic reynolds stress models for anisotropic wall-bounded flows. Progress in Flight Physics, 2012, 3: 89-104. DOI: $10.1051 /$ eucass/201203089.

[10] Langtry R., Menter F., Transition modeling for general CFD applications in aeronautics. 43rd AIAA Aerospace Science Meeting and Exhibit, American Institute of Aeronautics and Astronautics, 2005, AIAA 2005-522. DOI: $10.2514 / 6.2005-522$.

[11] Petersen A., Doerffer P., Flaszynski P., Szwaba R., Piotrowicz M., Kaczynski P., Tartinville B., Hirsch C., WP-4 Internal Flows-Turbine. Transition Location Effect on Shock Wave Boundary Layer Interaction, 2021, pp. 297-346. DOI: 10.1007/978-3-030-47461-4_5.

[12] Souverein L., Debiève J.F., Effect of air jet vortex generators on a shock wave boundary layer interaction. Experiments in Fluids, 2010, 49: 1053-1064. DOI: $10.1007 / \mathrm{s} 00348-010-0854-8$.

[13] Flaszynski P., Doerffer P., Szwaba R., Numerical simulations of transonic flow with film cooling and jet vortex generators. Proceedings of 9th European Turbomachinery Conference, Istanbul, March, 2011, 2: 1615-1625. 